\title{
Nonequilibrium phases in hybrid arrays with flux qubits and nitrogen-vacancy centers
}

\author{
Thomas Hümmer, ${ }^{1,2}$ Georg M. Reuther, ${ }^{2}$ Peter Hänggi, ${ }^{2}$ and David Zueco ${ }^{1,2,3}$ \\ ${ }^{1}$ Instituto de Ciencia de Materiales de Aragón y Departamento de Física de la Materia Condensada, CSIC-Universidad de Zaragoza, \\ E-50009 Zaragoza, Spain \\ ${ }^{2}$ Institut für Physik, Universität Augsburg, Universitätsstraße 1, D-86135 Augsburg, Germany \\ ${ }^{3}$ Fundación ARAID, Paseo María Agustín 36, E-50004 Zaragoza, Spain
}

(Received 8 March 2012; published 25 May 2012)

\begin{abstract}
We propose a startling hybrid quantum architecture for simulating a localization-delocalization transition. The concept is based on an array of superconducting flux qubits which are coupled to a diamond crystal containing nitrogen-vacancy centers. The underlying description is a Jaynes-Cummings lattice in the strong-coupling regime. However, in contrast to well-studied coupled cavity arrays, the interaction between lattice sites is mediated here by the qubit rather than by the oscillator degrees of freedom. Nevertheless, we point out that a transition between a localized and a delocalized phase occurs in this system as well. We demonstrate the possibility of monitoring this transition in a nonequilibrium scenario, including decoherence effects. The proposed scheme allows the monitoring of localization-delocalization transitions in Jaynes-Cummings lattices by use of currently available experimental technology. Contrary to cavity-coupled lattices, our proposed recourse to stylized qubit networks facilitates (i) investigating localization-delocalization transitions in arbitrary dimensions and (ii) tuning the intersite coupling in situ.
\end{abstract}

DOI: 10.1103/PhysRevA.85.052320

PACS number(s): 03.67.Lx, 05.30.Rt, 42.50.Ct, 85.25.Cp

\section{INTRODUCTION}

In recent years, a variety of novel experimental approaches have enabled tests of fundamental quantum physics, such as superpositions, entanglement, tunneling, or quantum phase transitions in artificial devices. Prominent examples hereof are quantum circuits, quantum dots, or optical lattices [1,2]. Apart from their fundamental relevance, quantum technologies allow realizing quantum information processors that bring along the potential of carrying out specific tasks at exponentially reduced computation time [3]. Furthermore, quantum simulators of the Feynman type [4,5], employed to simulate the dynamics of one quantum system by means of another one, are a pivotal example of quantum speedup as compared to a classical computer. While both systems share the same dynamics, the simulator offers far more configurability and is better accessible for a measurement.

Generally, the possibility of observing quantum effects strongly depends on the coherence properties of the underlying system. With regard to superconducting circuit qubits, the advantages of versatile manufacturing, detection, and manipulation are paid for at the price of quite high decoherence rates as compared to trapped ions or spin qubits. One way out is given by the recently emerging field of hybrid systems [6-14]. The main motivation for building hybrid systems is to combine two advantages: the addressability of artificial quantum circuits and the long coherence times of elemental systems, such as nitrogen-vacancy (NV) centers in diamonds or in polar molecules. Usually these hybrid systems are motivated by using the natural spins for building quantum memories.

In this paper, we point out an alternative application for the exploration of many-body physics, such as quantum phase transitions. In particular, we investigate the localized and delocalized phases that occur in Hubbard-like models, such as the Jaynes-Cummings (JC) lattice [15-18]. In the localized phase, excitations are localized at individual lattice sites, whereas they are delocalized across the lattice in the delocalized phase. We propose an intriguingly simple layout for simulating a JC lattice: We use the combination of an already experimentally well-proven flux qubit array together with a single large NV-center crystal. By means of numerical studies, we corroborate that this system exhibits localized and delocalized phases. Finally, we demonstrate that these phases can be identified by monitoring the signatures of the nonequilibrium system dynamics in the presence of decoherence and dissipation upon employing experimentally accessible parameters.

\section{HYBRID QUBIT-RESONATOR MODEL}

As the elementary unit for the JC array, we propose a hybrid combination of a flux qubit and an ensemble of independent spins, given here by the NV centers in a diamond $[19,20]$. As illustrated in Fig. 1(a), the spin crystal is placed in proximity of a qubit loop. The spin-qubit Zeeman interaction is mediated by the magnetic field that stems from the qubit's persistent currents. A weak external field splits the spin degeneracy so as to shift one spin transition into resonance with the qubit's transition frequency $\omega_{q}$. Reducing the spin to its two lowest levels with mutual energy spacing $\omega_{c}$ [see Fig. 1(b)] and applying the rotating wave approximation (RWA), we can express the Hamiltonian of an ensemble of $N$ spins coupled to a single flux qubit at its degeneracy point as $(\hbar=1)$

$$
H_{q+s}=\omega_{q} \sigma^{+} \sigma^{-}+\omega_{c} \sum_{k}^{N} \tau_{k}^{+} \tau_{k}^{-}+\sum_{k}^{N}\left(g_{k} \tau_{k}^{+} \sigma^{-}+\text {H.c. }\right)
$$

Here, $\sigma^{ \pm}=\sigma^{x} \pm i \sigma^{y}$ and $\tau_{k}^{ \pm}=\tau_{k}^{x} \pm i \tau_{k}^{y}$ are raising and lowering operators with respect to the qubit's $(\sigma)$ and the spins' $\left(\tau_{k}\right)$ Pauli matrices. The coupling strengths $g_{k}$ between the qubit and the individual spins are proportional to the magnitude of the qubit's field at the spin positions [19,20]. Assuming the 
(a)

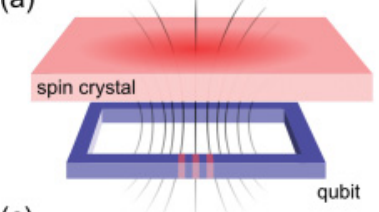

(b)

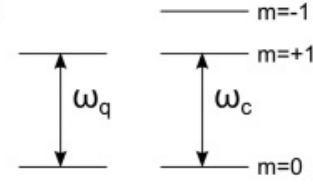

(c)

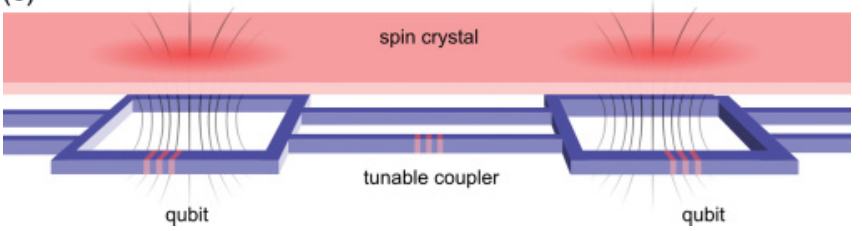

FIG. 1. (Color online) (a) Schematic of a single flux qubit coupled to a diamond crystal with NV centers embedded. (b) Energy diagram for the qubit with level splitting $\omega_{q}$ and an NV center. By applying an external magnetic field, the level with spin projection $m= \pm 1$ becomes resonant with the upper qubit level. (c) The JC array with tunable qubit-qubit coupling. Here, adjacent qubits are connected via auxiliary tunable coupler qubits. Each of the qubits couples to spatially separated regions of the crystal. The coupler qubit does not couple to the spins because it is far detuned from the qubits and consequently from the NV spins as well.

case of a large spin ensemble with low polarization, i.e., close to its ground state, we introduce a collective operator: i.e., with $g=\left(\sum_{k}^{N}\left|g_{k}\right|^{2}\right)^{1 / 2}$ we set $a^{\dagger}=g^{-1} \sum_{k}^{N} g_{k} \tau_{k}^{+}$together with its Hermitian conjugate $a$, yielding approximately the bosonic commutation relation, $\left[a, a^{\dagger}\right] \cong 1$ (see Appendix A). Thus, we interpret the ensemble as an effective bosonic mode and arrive at the effective Jaynes-Cummings model, reading as

$$
H_{\mathrm{JC}}=\omega_{q} \sigma^{+} \sigma^{-}+\omega_{c} a^{\dagger} a+g\left(a^{\dagger} \sigma^{-}+a \sigma^{+}\right) .
$$

It describes a collective harmonic oscillator mode being coupled to a two-level system with the interaction strength $g$. The collective coupling $g$ is enhanced by a factor of $\sqrt{N}$ compared to the root mean square of the individual couplings $g_{k}$ (see Appendix A). Recent experiments achieved coupling strengths as strong as $g \approx 2 \pi \times 35 \mathrm{MHz}$ [21].

\section{THE JAYNES-CUMMINGS LATTICE WITH QUBIT-QUBIT COUPLING}

A general advantage of superconducting circuits is their scalability and the rich variety of coupling mechanisms that can be implemented on a chip. In particular, arrays of flux qubits with tunable coupling strength between individual qubits have been realized using a SQUID or ancilla flux qubit [22-26]. In recent experiments manipulating coupling strengths in situ and the engineering of various types of circuit connectivity have become feasible [27-31]. In this work, we restrict ourselves to a chain of qubits with a tunable nearest neighbor interaction. This array of qubits can be readily turned into a JC lattice of coupled qubit-oscillator systems by putting one NV-center crystal on top, as sketched with Fig. 1(c). As argued above, the spin crystal adds an effective harmonic oscillator degree of freedom to each site of the array. Apart from the possibility to tune the coupling between the sites of the lattice, the most appealing aspect of this hybrid architecture is simplicity. Furthermore, the harmonic oscillators in the form of the spin crystal exhibit excellent coherence properties, homogeneous transition frequencies, and coupling strengths, all implemented here within a reduced geometric dimension as compared to coplanar waveguide resonators.

For well-separated qubits, we can neglect their mutual inductance as well as the cross coupling of one qubit to the spin ensemble of another site, being even one order of magnitude smaller. This JC lattice with $M$ sites is thus described by

$$
H_{\mathrm{JCL}}=\sum_{j}^{M} H_{\mathrm{JC}, j}+J \sum_{j}^{M-1}\left(\sigma_{j}^{+} \sigma_{j+1}^{-}+\text {H.c. }\right) \text {, }
$$

with the single-site Hamiltonians $H_{\mathrm{JC}, j}$ given in Eq. (2). Here, $J$ denotes the uniform qubit-qubit coupling strength and the operators $\sigma_{j}^{ \pm}$describing the creation and annihilation of a qubit excitation at the $j$ th site.

A subtle but salient difference between our model and previously studied JC lattices is in the interaction mechanism between individual lattice sites. While we propose an intersite coupling mediated by the qubits, previous works have dealt with the complementary approach where the lattice sites interact via the oscillator degrees of freedom, as in coupled cavities [15-18,32,33] or in superconducting resonators [34,35]. In the latter case, the coupling part of the JC-lattice Hamiltonian (3) assumes the form $a_{j}^{\dagger} a_{j+1}+$ H.c.

\section{EQUILIBRIUM PROPERTIES OF THE JC LATTICE}

For Bose-Hubbard-like models the occurrence of a quantum phase transition between localized and delocalized phases has been extensively studied [36]. Analogous transitions have been investigated with polaritons in JC lattices [15-18,32,34,35]. Here, the term polariton refers to the eigenstates $|n, \pm\rangle$ of the single-site Hamiltonian $H_{\text {JC }}$ [Eq. (2)] (see Appendix B). The excitation number $n$, being the eigenvalues of the operator $\mathcal{N}=a^{\dagger} a+\sigma^{+} \sigma^{-}$, are conserved due to $\left[H_{\mathrm{JC}}, \mathcal{N}\right]=0$. Similarly, the full JC lattice Hamiltonian (3) conserves the total number of excitations in the lattice. The ground state $|0\rangle$ has no excitations $n=0$, while the states $|n, \pm\rangle(n>0)$ are each twofold degenerate with respect to $\mathcal{N}$. If the qubit and the resonator are in resonance, $\omega_{c}=\omega_{q}$, the polaritonic states are symmetric $(+)$ and antisymmetric $(-)$ superpositions $|n, \pm\rangle=$ $(|n-1\rangle|\uparrow\rangle \pm|n\rangle|\downarrow\rangle) / \sqrt{2}$ of the oscillator Fock states $|n\rangle$ and the qubit ground $(|\downarrow\rangle)$ and excited $(|\uparrow\rangle)$ states, respectively.

The localization-delocalization transition we consider in this paper takes place for the lowest energy state in the subspace with one average excitation per site $[17,18]$. As we argue with Appendix D, for weak intersite coupling $J$, no interconversion between the + and - polaritons occurs $[17,34]$. Therefore, in order to obtain analytical estimates, we can neglect $|n,+\rangle$ polaritons and restrict our studies to $|n,-\rangle$ polaritons which are lower in energy. We then introduce the "effective repulsion" $\delta=E_{|2,-\rangle}-2 E_{|1,-\rangle}$, i.e.,

$$
\delta=-\sqrt{2 g^{2}+\frac{\Delta^{2}}{4}}+2 \sqrt{g^{2}+\frac{\Delta^{2}}{4}}-\frac{\Delta}{2} .
$$

This positive-valued repulsion increases with the qubitoscillator coupling strength $g$ and decreases with the detuning $\Delta=\omega_{q}-\omega_{c}$. It measures the extra energy needed to insert two polaritons into a single site as compared to distributing 
them across two sites. Thus, a large repulsion promotes an even distribution of excitations over the lattice sites. In this case, the system eigenstate is approximately given by a product of the local single-site eigenstates $|1,-\rangle_{j}$.

By contrast, a large intersite coupling quantified by $J$ favors delocalized excitations, i.e., momentum eigenstates that are given by a superposition of product states, each with different $n$. Thus, modifying $J$ or the repulsion (e.g., by means of $\Delta$ ) one ends up in two extreme regimes: the localized or the delocalized phase.

As stated at the beginning of this section, the Hamiltonian (3) conserves the total number of excitations. Therefore, the fluctuation of the excitation number in a particular lattice site, $\operatorname{var}\left(\mathcal{N}_{j}\right)=\left\langle\mathcal{N}_{j}^{2}\right\rangle-\left\langle\mathcal{N}_{j}\right\rangle^{2}, \quad$ is used as an order parameter in JC lattices [34]. For $\operatorname{var}\left(\mathcal{N}_{j}\right)=0$, the excitations are trapped, and the system is in a localized phase. By contrast, large fluctuations indicate the delocalized phase.

In order to investigate the transition between the localized and the delocalized phases numerically, we calculate $\operatorname{var}\left(\mathcal{N}_{j}\right)$ for a setup with two sites. As indicated in the main panel of Fig. 2(a), a transition between the two different regimes characterized by zero and finite $\operatorname{var}\left(\mathcal{N}_{j}\right)$ occurs upon a change of $\Delta$ or $J$. This two-site setup already exhibits the same qualitative features as longer arrays of finite length, as we corroborate in the side panels of Fig. 2(a). There, we compare the variance for arrays with two or more sites by means of two cross sections through the main panel for fixed values of $\Delta$ and $J$, respectively. Thus, the elementary twosite setup-readily feasible with present-day experimental techniques - already allows for a good qualitative estimate of the transition properties of a JC array. Experimental feasibility will be further discussed in Sec. VI

To gain analytical insight, we express the coupling between the individual sites in terms of the relevant polaritonic basis states $|n,-\rangle_{j}$. In doing so, we can approximate $\sigma_{j}^{+}=$ $\sum_{n}^{\infty} s_{n,--}|n+1,-\rangle_{j}\left\langle n,-\left.\right|_{j}\right.$, where the coefficients $s_{n,--}$ depend on $\Delta, g$, and $n$ and their explicit form is detailed in Appendix C. Thus, two sites initially in the state $|1,-\rangle$ are coupled with the effective strength (see Appendix C)

$$
J_{\text {eff }}=J s_{0,--} s_{1,--} .
$$

In the lower panel of Fig. 2(b), we compare $J_{\text {eff }}$ to the effective repulsion strength $\delta$ [Eq. (4)], both plotted as functions of $\Delta$ at fixed $J$. We find that the observed crossing point of $J_{\text {eff }}$ and $\delta$ closely matches the location of the localization-delocalization transition.

Furthermore, we compare our results for a JC array with qubit-qubit (QQ) coupling to a similar setup with cavity-cavity (CC) coupling, i.e., an array in which the individual sites interact via their oscillator degrees of freedom $J\left(a_{j}^{\dagger} a_{j+1}+\right.$ h.c). For this latter scenario, we find that the transition to the delocalized phase already occurs at smaller $\Delta$ [see Fig. 2(b), top panel]. As in the QQ-coupled case we calculate $J_{\text {eff }}$ via the relevant polaritonic basis states and indeed find a larger effective intersite interaction that hence explains the observed transition point. The interested reader can check the explicit coupling coefficients in Appendix C. With increasing detuning, the $|n,-\rangle_{j}$ polaritons become more and more bosonic, i.e., only the oscillator degree of freedom is excited, $|n,-\rangle_{j} \approx|\downarrow\rangle_{j}|n\rangle_{j}$.

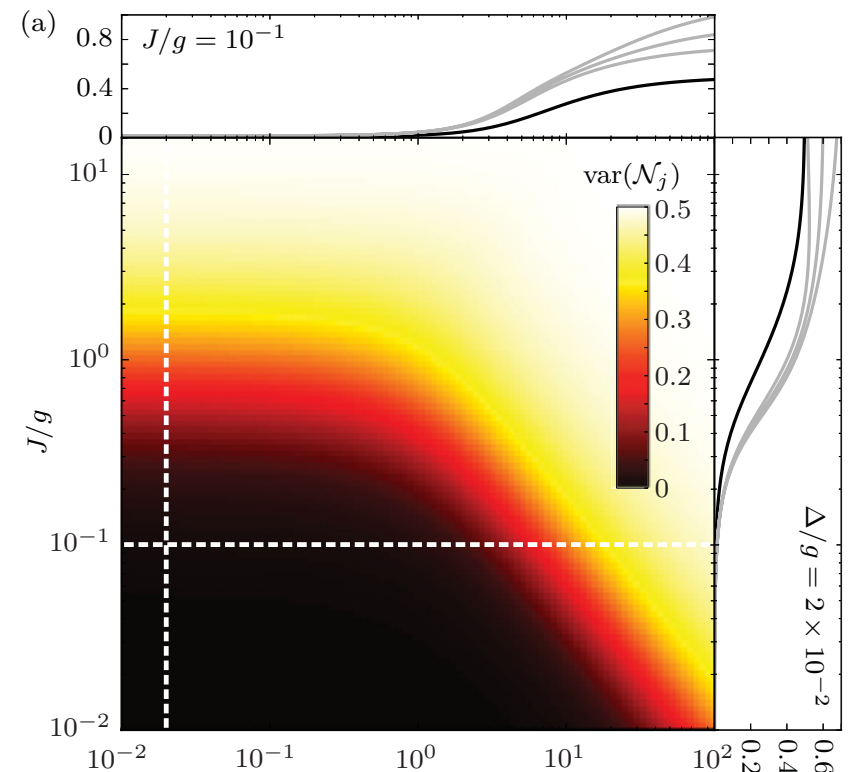

(b)

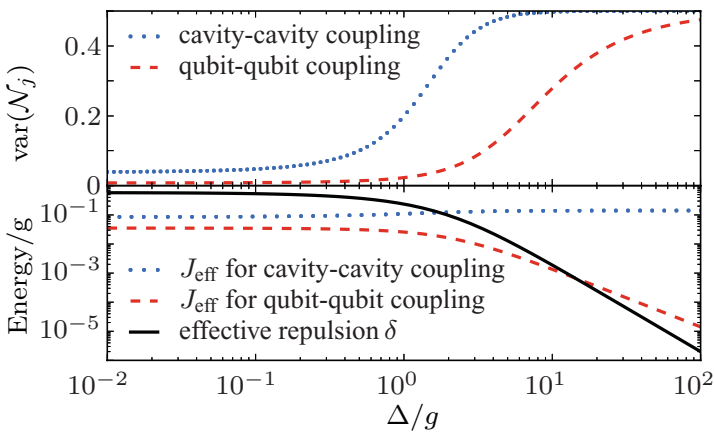

FIG. 2. (Color online) Transition between the localized and delocalized phases in a qubit-coupled JC array. (a) Fluctuations of the number of excitations at a certain site $\operatorname{var}\left(\mathcal{N}_{j}\right)$ for a two-site setup (both sites $j=1,2$ yield the same plot) as a function of the intersite coupling $J$ and the qubit-ensemble detuning $\Delta$. The dark shaded region indicates that the system is in the localized phase, while the brighter areas are related to large fluctuations, i.e., the delocalized phase. The two side panels depict a horizontal cut along $J=0.1 \mathrm{~g}$ and a vertical cut along $\Delta=2 \times 10^{-2} \mathrm{~g}$ (i.e., very close to qubit-oscillator resonance), respectively. There, the solid black curves depict $\operatorname{var}\left(\mathcal{N}_{j}\right)$ for the two-site setup as in the central panel. For comparison, we have included the fluctuation characteristics $\operatorname{var}\left(\mathcal{N}_{j}\right)$ for longer JC arrays with $N=3-5$ sites in ascending order, where $j$ denotes a central site of the array. (b) Comparison of QQ- and CC-coupled chains. In the latter, the transition occurs at lower detunings due to higher effective coupling of polaritons between adjacent sites. This can be seen in the lower plot: Changing the detuning affects both the effective repulsion $\delta$, as well as the effective coupling $J_{\text {eff }}$. The transition occurs when $\delta$ and $J_{\text {eff }}$ cross.

This allows for a simple explanation of the different trends of $J_{\text {eff }}$ in Fig. 2(b) when increasing the detuning. In a qubit-coupled array, the bosonic excitations must hop via the route oscillator-qubit-qubit-oscillator to reach the next lattice site; therefore $J_{\text {eff }}$ is small. By contrast, in the cavity-coupled setup the bosonic excitations can hop directly to the next-site oscillator, and $J_{\text {eff }}$ is, therefore, larger when the excitations are purely bosonic. 

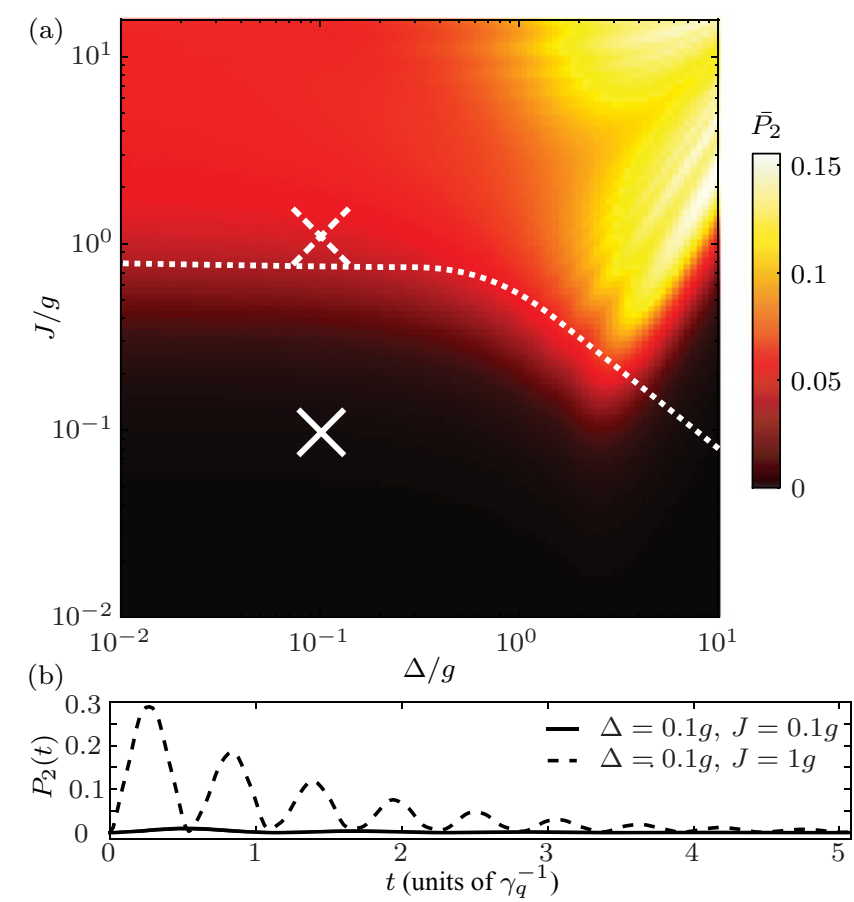

FIG. 3. (Color online) Nonequilibrium signature of the phase transition. (a) Time-averaged probability $\bar{P}_{2}$ to find two excitations in a single site. The coupling is assumed as $g=2 \pi \times 10 \mathrm{MHz}$, and the decay rates of qubits and oscillators are $\gamma_{\mathrm{q}}=2 \pi \times 1 \mathrm{MHz}$ and $\gamma_{\mathrm{c}}=2 \pi \times 0.1 \mathrm{MHz}$, respectively. We choose the integration time as $T=5 \gamma_{\mathrm{q}}^{-1}$. The dotted line marks the boundary $\left(1 / 2 \max \left\{\operatorname{var}\left(\mathcal{N}_{j}\right)\right\}\right)$ where the phase transition occurs in the equilibrium case of Fig. 2. (b) Time evolution of $P_{2}$ in two exemplary points in the delocalized [dashed line in (b) and dashed cross in (a)] and localized phase [solid line in (b) and solid cross in (a)], respectively.

\section{NONEQUILIBRIUM DYNAMICS}

The characterization of localized and delocalized phases at equilibrium is helpful in exploring the physics in $\mathrm{JC}$ lattices with qubit-qubit coupling and contrasting it with JC lattices that interact with a cavity-cavity coupling. However, the assumption of staying in the subspace with one mean excitation per site is not completely realistic in practice. In particular, nonequilibrium processes, such as dissipation and decoherence, are crucial in solid state devices. Next we point out that the signatures of the localization-delocalization transition remain preserved even in the presence of dissipation. A corresponding measurement only requires state preparation and qubit readout.

We model dissipation for both the qubit and spin ensemble by means of a quantum master equation, which for the JC lattice assumes the form (at zero temperature) [37,38]

$$
\dot{\rho}(t)=-i\left[H_{\mathrm{JCL}}, \rho\right]+\sum_{j}\left(\gamma_{c} L_{a_{j}}[\rho(t)]+\gamma_{q} L_{\sigma_{j}^{-}}[\rho(t)]\right) .
$$

The Lindblad dissipators $L_{O}$ act on the density operator $\rho$ as $L_{O}[\rho]=O \rho O^{\dagger}-\frac{1}{2}\left(O^{\dagger} O \rho+\rho O^{\dagger} O\right)$. The operators $O=$ $\left\{\sigma_{j}^{-}, a_{j}\right\}$ describe the system-bath coupling of the $j$ th qubit and oscillator, respectively, while $\gamma_{q}$ and $\gamma_{c}$ are the associated, uniform decoherence rates.
The system is initially prepared with one $|1,-\rangle$ polariton in each site. Calculating the system dynamics numerically, we obtain the time-dependent probability $P_{2}(t)=\operatorname{Tr}\left\{\Pi_{2} \rho(t)\right\}$ of finding two excitations in one site, where $\Pi_{2}=|2\rangle|\downarrow\rangle\langle g|\langle 2|+$ $|1\rangle|\uparrow\rangle\langle e|\langle 1|$. If the system is in the localized phase, we expect $P_{2}(t)$ to remain close to zero. By contrast, in the the delocalized phase, $P_{2}(t)$ reaches finite values over time. This behavior is depicted in Fig. 3(b). Here, it is also visible that the system evolves eventually into its ground state due to decoherence.

In order to quantify the phase we introduce the averaged probability

$$
\bar{P}_{2}=\frac{1}{T} \int_{0}^{T} d t P_{2}(t) .
$$

In order to take into account the dynamics before relaxation into the ground state dominates, the integration time should fulfill $T \gg J_{\text {eff }}^{-1}$ but $T \lesssim \min \left\{\gamma_{c}^{-1}, \gamma_{q}^{-1}\right\}$. Figure 3(a) depicts $\bar{P}_{2}$ as a function of both the hopping parameter $J$ and the detuning $\Delta$ similar to the equilibrium analysis. For comparison, the white dashed line marks the parameter regime where the phase transition occurs in the equilibrium case in Fig. 2. While we find a good agreement for small values of $\Delta$, the border between both phases is not resolved in the far-detuned limit where the effective intersite coupling strength $J_{\text {eff }}$ decreases below the decoherence rates $\left\{\gamma_{q}, \gamma_{c}\right\}$.

\section{EXPERIMENTAL FEASIBILITY}

We next address the feasibility of the JC array proposed in this paper. The case of strong coupling between an ensemble of NV centers $\left(\omega_{c} \approx 2 \pi \times 2.88 \mathrm{MHz}\right)$ and a flux qubit has previously been reported experimentally with coupling strengths up to $g \simeq 2 \pi \times 35 \mathrm{MHz}$ [21]. Thus, using our estimations, we can safely consider a coupling strength of $g \cong 2 \pi \times 10 \mathrm{MHz}$. On the other hand, the experimental accessible tunable qubit-qubit couplings are between $2 \pi \times 1 \mathrm{MHz} \leqslant J \leqslant 2 \pi \times 100 \mathrm{MHz}$ $[23,25,26]$. All together, this sets the operation range to $0.1 \leqslant J / g \leqslant 10$. We next consider the decay rates of the involved subsystems. Realistic values for the qubit decay rates are $\gamma_{q}=2 \pi \times 1 \mathrm{MHz}$ (i.e., $\gamma_{q} / g=0.1$ ) and for the spin decay rates are $\gamma_{c} \leqslant 2 \pi \times 0.1 \mathrm{MHz}$ (i.e., $\gamma_{c} / g \leqslant 0.01$ ).

These values were used to obtain the plots in Fig. 3. Therefore, current technology allows for monitoring both phases. Finally, a readout of the number of excitations at a specific site can be performed by measuring the qubit dynamics.

\section{CONCLUSIONS}

In summary, we have introduced a JC lattice based on a hybrid combination of flux qubits and NV centers. In contrast to JC lattices based on coupled cavities or superconducting resonators, the harmonic oscillator degree of freedom ("cavity") is smaller in size than the qubit ("atom"). This allows one to couple the individual JC sites via the qubits instead of the harmonic oscillators. We have argued that similarly to cavitycoupled JC lattices a localization-delocalization transition can be observed in these qubit-coupled JC lattices. Even though localization-delocalization transitions in JC lattices have been proposed theoretically some time ago, they could not be 
observed in an experiment yet. Our proposal relies on a straightforward modification of already realized flux qubit arrays by simply mounting a single NV-center crystal on top. This minimal modification of a common setup opens the possibility of studying many-body phenomena in strongly coupled hybrid architectures within state-of-the-art experimental technology. Apart from its simplicity, further advantages are the possibility to investigate localization-delocalization transitions in arbitrary (even fractal) dimensions and to tune the intersite coupling in situ by using common techniques for building flux qubit networks.

\section{ACKNOWLEDGMENTS}

We acknowledge enlightening discussions with M. Hartmann at TUM. This work was supported by Spanish MICINN Projects No. FIS2011-25167 and No. CSD2007-046 Nanolight.es, the European PROMISCE, and the DFG via the Collaborative Research Center SFB 631 and the Nanosystems Initiative Munich (NIM).

\section{APPENDIX A: COLLECTIVE MODES}

Here we outline the approximation that allows us to express the spin ensemble by a collective bosonic operator. With homogeneous coupling for all the spins, one can express the spins by a collective angular momentum operator and then apply a Holstein-Primakoff approximation to yield a bosonic operator. However, for inhomogeneous couplings this is not readily possible because the collective operator does not fulfill angular momentum algebra. Nevertheless, we can arrive at collective bosonic operators: We start with the Hamiltonian of spins (inhomogeneously) coupled to a qubit with the individual coupling strength $g_{k}$ :

$$
H=\omega_{q} \sigma^{+} \sigma^{-}+\omega_{c} \sum_{k}^{N} \tau_{k}^{+} \tau_{k}^{-}+\sum_{k}^{N}\left(g_{k} \sigma^{-} \tau_{k}^{+}+\text {H. с. }\right) \text {. }
$$

Here, $\tau_{k}^{ \pm}$are the Pauli raising and lowering operators of the spins in the ensemble and $\sigma^{ \pm}$the ones of the qubit. The spins are taken to have the homogeneous energy splitting $\omega_{c}$ and the qubit the splitting $\omega_{q}$. We next introduce the collective operator

$$
a^{\dagger}=\frac{1}{\sqrt{N} \bar{g}} \sum_{k}^{N} g_{k} \tau_{k}^{+}
$$

where $\bar{g}$ is the root mean square of the individual couplings, $\bar{g}^{2} \equiv \sum_{k}\left|g_{k}\right|^{2} / N$. In the low polarization limit, where almost all spins are in the ground state, it follows that these operators approximately fulfill bosonic commutation relations. To validate this we calculate the commutator

$$
\begin{aligned}
{\left[a, a^{\dagger}\right] } & =\frac{1}{N \bar{g}^{2}} \sum_{k l} g_{k}^{*} g_{l}\left[\tau_{k}^{-}, \tau_{l}^{+}\right] \\
& =\frac{1}{N \bar{g}^{2}} \sum_{k}\left|g_{k}\right|^{2}\left[\tau_{k}^{-}, \tau_{k}^{+}\right] \\
& =\frac{1}{N \bar{g}^{2}} \sum_{k}\left|g_{k}\right|^{2}\left(\mathbb{I}_{k}-2 \tau_{k}^{+} \tau_{k}^{-}\right) .
\end{aligned}
$$

Inserting the definition of $\bar{g}$ yields

$$
\left[a, a^{\dagger}\right]=1-\frac{2}{N \bar{g}^{2}} \sum_{k}\left|g_{k}\right|^{2} \tau_{k}^{+} \tau_{k}^{-} .
$$

For states with only a few spins excited, the second term is $\ll 1$ and thus $a^{\dagger}$ and $a$ obey approximately bosonic commutation relations,

$$
\left[a, a^{\dagger}\right] \approx 1
$$

Using these collective operators the coupling term of Hamiltonian (1) becomes

$$
\sqrt{N} \bar{g}\left(\sigma^{+} a+\sigma^{-} a^{\dagger}\right) .
$$

If we start with the spin ensemble in the ground state $|0\rangle$ and with the qubit excited, Rabi oscillations can transform excitations into the ensemble that assume the form of generalized Dicke states

$$
\begin{aligned}
a^{\dagger}|0\rangle & =\frac{1}{\sqrt{N} \bar{g}} \sum_{k}^{N} g_{k} \tau_{k}^{+}|0\rangle \\
& =\frac{1}{\sqrt{N} \bar{g}} \sum_{k}^{N} g_{k}\left|0_{1} \cdots 1_{k} \cdots 0_{N}\right\rangle \equiv|1\rangle .
\end{aligned}
$$

Here $\left|0_{1} \cdots 1_{k} \cdots 0_{N}\right\rangle$ denotes a state where all spins are in the ground state except the $k$ th spin. Higher excited states are defined by

$$
|n\rangle=\frac{1}{\sqrt{n !}}\left(a^{\dagger}\right)^{n}|0\rangle .
$$

If we restrict our Hilbert space to the set of states with this symmetry, we can express $\sum_{k}^{N} \tau_{k}^{+} \tau_{k}^{-}$in the first part of Hamiltonian (A1) by collective operators as well. To this end, we show that $\sum_{k}^{N} \tau_{k}^{+} \tau_{k}^{-}$gives the number of collective excitations for state $|0\rangle$,

$$
\sum_{k}^{N} \tau_{k}^{+} \tau_{k}^{-}|0\rangle=0|0\rangle
$$

and we use induction to show that if it is true for $|n\rangle$ it is also true for $|n+1\rangle$ :

$$
\begin{aligned}
\sum_{k}^{N} \tau_{k}^{+} \tau_{k}^{-}|n+1\rangle= & \sum_{k}^{N} \tau_{k}^{+} \tau_{k}^{-} \frac{1}{\sqrt{n+1}} a^{\dagger}|n\rangle \\
= & \frac{1}{\sqrt{n+1}} \sum_{k}^{N} \tau_{k}^{+} \tau_{k}^{-} \frac{1}{\sqrt{N} \bar{g}} \sum_{l}^{N} g_{l} \tau_{l}^{+}|n\rangle \\
= & \frac{1}{\sqrt{n+1}} \frac{1}{\sqrt{N} \bar{g}}\left(\sum_{l}^{N} g_{l} \tau_{l}^{+} \sum_{k}^{N} \tau_{k}^{+} \tau_{k}^{-}|n\rangle\right. \\
& \left.+\sum_{k, l}^{N} g_{l} \tau_{k}^{+}\left[\tau_{k}^{-}, \tau_{l}^{+}\right]|n\rangle\right) \\
= & n|n+1\rangle+1|n+1\rangle=(n+1)|n+1\rangle .
\end{aligned}
$$

In the next to last step in Eq. (A9), we insert the relation: $\sum_{k}^{N} \tau_{k}^{+} \tau_{k}^{-}|n\rangle=n|n\rangle$, valid by induction. Besides, we use the Pauli matrices conmutation relation: $\left[\tau_{k}^{-}, \tau_{l}^{+}\right]=$ 
$\delta_{k l}\left(\mathbb{I}_{k}-2 \tau_{k}^{+} \tau_{k}^{-}\right)$and notice that $\tau_{k}^{+} \tau_{k}^{+}=0$. Therefore, we can write in the subspace of the collective excitations $|n\rangle$,

$$
\sum_{k}^{N} \tau_{k}^{+} \tau_{k}^{-}=a^{\dagger} a
$$

In conclusion, restricting ourselves to the Hilbert space of the states $|n\rangle$, the initial Hamiltonian (A1) can be recast as

$$
H=\omega_{q} \sigma^{+} \sigma^{-}+\omega_{c} a^{\dagger} a+\bar{g} \sqrt{N}\left(\sigma^{+} a+\sigma^{-} a^{\dagger}\right) .
$$

Besides, in the low polarization limit $a^{\dagger}$ and $a$ obey approximately bosonic commutation relations. Finally, it follows that the collective coupling is enhanced by a factor of $\sqrt{N}$ as compared to the root mean square of the couplings to the individual spins.

\section{APPENDIX B: THE POLARITONIC BASIS}

The eigenstates of a single JC Hamiltonian (A11) are the socalled polaritons and are denoted by $|n, \pm\rangle_{j}$. Here, $n$ describes the number of excitations at a site (sum of qubit and bosonic excitations), while the sign defines the polariton "species." We now use these polaritons to express the Hamiltonian of the whole JC chain.

Using the polariton eigenstates $|n, \pm\rangle_{j}$ at site $j$, the individual uncoupled JC Hamiltonians are diagonal,

$$
H_{j}^{\mathrm{JC}}=\sum_{n=0}^{\infty} \sum_{\alpha= \pm} E_{n, \alpha}|n, \alpha\rangle_{j}\left\langle n,\left.\alpha\right|_{j}\right.
$$

The individual Jaynes-Cummings energies $E_{n, \alpha}$ at a certain site are thus given by

$$
E_{n, \pm}=n \omega_{c}+\frac{\Delta}{2} \pm \sqrt{n g^{2}+\Delta^{2} / 4},
$$

With the detuning $\Delta=\omega_{q}-\omega_{c}$. Note that the $|n,-\rangle$ polaritons are lower in energy than their $|n,+\rangle$ counterpart. The eigenstates are

$$
\begin{gathered}
|n,-\rangle \equiv \cos \theta_{n}|n, \downarrow\rangle-\sin \theta_{n}|n-1, \uparrow\rangle, \\
|n,+\rangle \equiv \sin \theta_{n}|n, \downarrow\rangle+\cos \theta_{n}|n-1, \uparrow\rangle,
\end{gathered}
$$

and $|0\rangle$ with $E_{0}=0$ where the mixing angle $\theta_{n}$ is defined as

$$
\theta_{n}=\frac{1}{2} \arctan \left(\frac{g \sqrt{n}}{\Delta / 2}\right)
$$

With increasing detuning the $|n,-\rangle$ polaritons resemble more and more pure bosonic excitations, $|n,-\rangle \approx|n\rangle|\downarrow\rangle$, while the $|n,+\rangle$ polaritons exhibit an excitation in the qubit, $|n,+\rangle \approx$ $|n-1\rangle|\uparrow\rangle$.

\section{APPENDIX C: HOPPING TERM IN THE POLARITONIC BASIS}

Next we need to express the intersite-hopping terms,

$$
H_{j}^{\mathrm{hop}}=J\left(\sigma_{j}^{+} \sigma_{j+1}+\text { H.c. }\right)
$$

in the local JC-basis as well. The operator $\sigma_{j}^{+}$takes the form

$$
\sigma_{j}^{+}=\sum_{n=0}^{\infty} \sum_{\alpha \beta= \pm} s_{n \alpha \beta}|n+1, \beta\rangle_{j}\left\langle n,\left.\alpha\right|_{j},\right.
$$

where the coefficients $s_{n \alpha \beta}$ are given by

$\left(\begin{array}{ll}s_{n--} & s_{n-+} \\ s_{n+-} & s_{n++}\end{array}\right)=\left(\begin{array}{ll}-\cos \theta_{n} \sin \theta_{n+1} & \cos \theta_{n} \cos \theta_{n+1} \\ -\sin \theta_{n} \sin \theta_{n+1} & \sin \theta_{n} \cos \theta_{n+1}\end{array}\right)$.

These coefficients depend on the coupling $g$ and the detuning $\Delta$ between the oscillator and the qubit via (B4). Note that the operator $\sigma_{j}^{+}$acts on a polariton state $|n, \alpha= \pm\rangle_{j}$ by transforming it to a linear combination of polaritons \pm with an additional excitation

$$
\sigma_{j}^{+}|n, \alpha\rangle_{j}=\sum_{\beta= \pm} s_{n \alpha \beta}|n+1, \beta\rangle_{j}
$$

The hopping term can now be rewritten in the polaritonic basis as

$$
\begin{aligned}
H_{j}^{\text {hop }}= & J \sum_{n n^{\prime}} \sum_{\alpha \alpha^{\prime}} \sum_{\beta \beta^{\prime}} s_{n \alpha \beta}|n+1, \beta\rangle_{j}\left\langle n,\left.\alpha\right|_{j}\right. \\
& \times s_{n^{\prime} \alpha^{\prime} \beta^{\prime}}\left|n^{\prime}, \alpha^{\prime}\right\rangle_{j+1}\left\langle n^{\prime}+1,\left.\beta^{\prime}\right|_{j+1}+\right.\text { H. c. }
\end{aligned}
$$

The hopping term, therefore, allows transitions between polaritons of different types on adjacent sites.

\section{Effective coupling $J_{\text {eff }}$ in the case of two sites}

For a two site array initially in the state $|11\rangle \equiv|1,-\rangle_{1} \otimes$ $|1,-\rangle_{2}$, we can derive an effective coupling constant between the two sites. Discarding transitions between different polariton species, the dynamics are spanned by $\{|11\rangle,|22\rangle\}$ with

$$
|22\rangle \equiv 1 / \sqrt{2}\left(|2,-\rangle_{1} \otimes|0,-\rangle_{2}+|2,-\rangle_{1} \otimes|0,-\rangle_{2}\right) .
$$

In this case the hopping term simply reduces to

$$
\left\langle 11\left|H_{j}^{\mathrm{hop}}\right| 22\right\rangle=J\left(s_{1--} s_{0--}+s_{1--} s_{0--}\right) .
$$

Therefore the effective coupling emerges as $J_{\text {eff }}=$ $J s_{1--} s_{0--}$.

\section{Effective cavity-cavity coupling}

As in the qubit-coupled case we rewrite the harmonic oscillator operators in the new basis

$$
a_{j}^{+}=\sum_{n=0}^{\infty} \sum_{\alpha \beta= \pm} t_{n \alpha \beta}|n+1, \beta\rangle_{j}\left\langle n,\left.\alpha\right|_{j},\right.
$$

where the coefficients $t_{n \alpha \beta}$ are given by

$$
\begin{aligned}
& t_{n--}=\cos \theta_{n} \cos \theta_{n+1} \sqrt{n+1}+\sin \theta_{n} \sin \theta_{n+1} \sqrt{n}, \\
& t_{n-+}=\cos \theta_{n} \sin \theta_{n+1} \sqrt{n+1}-\sin \theta_{n} \cos \theta_{n+1} \sqrt{n}, \\
& t_{n+-}=\sin \theta_{n} \cos \theta_{n+1} \sqrt{n+1}-\cos \theta_{n} \sin \theta_{n+1} \sqrt{n}, \\
& t_{n++}=\sin \theta_{n} \sin \theta_{n+1} \sqrt{n+1}+\cos \theta_{n} \cos \theta_{n+1} \sqrt{n} .
\end{aligned}
$$

For a two-site cavity coupled array, the effective coupling is finally given by $J_{\text {eff }}=J t_{1--} t_{0--}$. 


\section{APPENDIX D: CONSERVATION OF POLARITON TYPE}

In the previous Appendix we have seen that, generally, the coupling transfers polaritons from one site to another and may change the polariton type $( \pm)$ on the sites involved.

However, not all of these possible transitions actually have to occur. Transforming the coupling terms to the interaction picture with respect to the uncoupled Hamiltonian, i.e.,

$$
H_{0}=\sum_{j} H_{j}^{\mathrm{JC}}=\sum_{j, n, \alpha} E_{n, \alpha}|n, \alpha\rangle_{j}\left\langle n,\left.\alpha\right|_{j},\right.
$$

we detect that the individual terms of the coupling Hamiltonian, Eq. (C5), acquire time-dependent rotating phases

$$
\phi_{n n^{\prime} \alpha \alpha^{\prime} \beta \beta^{\prime}}(t)=e^{i t\left(E_{n+1, \beta}-E_{n, \alpha}\right)} e^{-i t\left(E_{n^{\prime}+1, \beta^{\prime}}-E_{n^{\prime}, \alpha^{\prime}}\right)} .
$$

We note that the overall frequency of the time dependent phase-given in terms of the energies of the states it is coupled to-broadly varies in magnitude. Thus, under the premise of a small intersite coupling $J$, we can identify fast oscillating terms and neglect those within a rotating wave approximation (RWA). Particularly, terms connecting states which are far apart in energy $(\gg J)$, and thus are rotating fast, are the ones that can be neglected.

This treatment is certainly useful in the case of large detuning or strong coupling strength $g$. Then, polaritons of different sign lie far apart in energy. Thus, their interconversion can safely be neglected and the initial polariton species $( \pm)$ is thus conserved.
[1] J. Q. You and F. Nori, Nature (London) 474, 589 (2011).

[2] I. Buluta, S. Ashhab, and F. Nori, Rep. Prog. Phys. 74, 104401 (2011).

[3] T. D. Ladd, F. Jelezko, R. Laflamme, Y. Nakamura, C. Monroe, and J. L. O'Brien, Nature (London) 464, 45 (2010).

[4] R. P. Feynman, Int. J. Theor. Phys. 21, 467 (1982).

[5] A. A. Houck, H. E. Türeci, and J. Koch, Nat. Phys. 8, 292 (2012).

[6] R. Amsüss, C. Koller, T. Nöbauer, S. Putz, S. Rotter, K. Sandner, S. Schneider, M. Schramböck, G. Steinhauser, H. Ritsch, J. Schmiedmayer, and J. Majer, Phys. Rev. Lett. 107, 060502 (2011).

[7] Y. Kubo, C. Grezes, A. Dewes, T. Umeda, J. Isoya, H. Sumiya, N. Morishita, H. Abe, S. Onoda, T. Ohshima, V. Jacques, A. Dréau, J. F. Roch, I. Diniz, A. Auffeves, D. Vion, D. Esteve, and P. Bertet, Phys. Rev. Lett. 107, 220501 (2011).

[8] Y. Kubo, F. R. Ong, P. Bertet, D. Vion, V. Jacques, D. Zheng, A. Dréau, J. F. Roch, A. Auffeves, F. Jelezko, J. Wrachtrup, M. F. Barthe, P. Bergonzo, and D. Esteve, Phys. Rev. Lett. 105, 140502 (2010).

[9] D. I. Schuster, A. P. Sears, E. Ginossar, L. DiCarlo, L. Frunzio, J. J. L. Morton, H. Wu, G. A. D. Briggs, B. B. Buckley, D. D. Awschalom, and R. J. Schoelkopf, Phys. Rev. Lett. 105, 140501 (2010).

[10] A. Imamoğlu, Phys. Rev. Lett. 102, 083602 (2009).

[11] P. Rabl, D. DeMille, J. M. Doyle, M. D. Lukin, R. J. Schoelkopf, and P. Zoller, Phys. Rev. Lett. 97, 033003 (2006).

[12] J. Verdu, H. Zoubi, C. Koller, J. Majer, H. Ritsch, and J. Schmiedmayer, Phys. Rev. Lett. 103, 043603 (2009).

[13] J. H. Wesenberg, A. Ardavan, G. A. D. Briggs, J. J. L. Morton, R. J. Schoelkopf, D. I. Schuster, and K. Molmer, Phys. Rev. Lett. 103, 070502 (2009).

[14] Z.-L. Xiang, S. Ashhab, J. Q. You, and F. Nori, arXiv:1204.2137.

[15] M. J. Hartmann, F. G. Brandao, and M. B. Plenio, Nat. Phys. 2, 849 (2006).

[16] A. D. Greentree, C. Tahan, J. H. Cole, and L. C. L. Hollenberg, Nat. Phys. 2, 856 (2006).

[17] D. G. Angelakis, M. F. Santos, and S. Bose, Phys. Rev. A 76, 031805 (2007).

[18] M. J. Hartmann, F. G. Brandao, and M. B. Plenio, Laser and Photon. Rev. 2, 527 (2008).

[19] D. Marcos, M. Wubs, J. M. Taylor, R. Aguado, M. D. Lukin, and A. S. Sorensen, Phys. Rev. Lett. 105, 210501 (2010).
[20] J. Twamley and S. D. Barrett, Phys. Rev. B 81, 241202 (2010).

[21] X. Zhu, S. Saito, A. Kemp, K. Kakuyanagi, S.-i. Karimoto, H. Nakano, W. J. Munro, Y. Tokura, M. S. Everitt, K. Nemoto, M. Kasu, N. Mizuochi, and K. Semba, Nature (London) 478, 221 (2011).

[22] K. Harrabi, F. Yoshihara, A. O. Niskanen, Y. Nakamura, and J. S. Tsai, Phys. Rev. B 79, 020507 (2009).

[23] T. Hime, P. A. Reichardt, B. L. T. Plourde, T. L. Robertson, C. Wu, A. V. Ustinov, and J. Clarke, Science 314, 1427 (2006).

[24] B. L. T. Plourde, J. Zhang, K. B. Whaley, F. K. Wilhelm, T. L. Robertson, T. Hime, S. Linzen, P. A. Reichardt, C. E. Wu, and J. Clarke, Phys. Rev. B 70, 140501 (2004).

[25] A. O. Niskanen, K. Harrabi, F. Yoshihara, Y. Nakamura, S. Lloyd, and J. S. Tsai, Science 316, 723 (2007).

[26] S. H. W. van der Ploeg, A. Izmalkov, A. M. van den Brink, U. Hübner, M. Grajcar, E. Ilichev, H. G. Meyer, and A. M. Zagoskin, Phys. Rev. Lett. 98, 057004 (2007).

[27] R. Harris, T. Lanting, A. J. Berkley, J. Johansson, M. W. Johnson, P. Bunyk, E. Ladizinsky, N. Ladizinsky, T. Oh, and S. Han, Phys. Rev. B 80, 052506 (2009).

[28] D. I. Tsomokos, S. Ashhab, and F. Nori, Phys. Rev. A 82, 052311 (2010).

[29] R. Harris, M. W. Johnson, T. Lanting, A. J. Berkley, J. Johansson, P. Bunyk, E. Tolkacheva, E. Ladizinsky, N. Ladizinsky, T. Oh et al., Phys. Rev. B 82, 024511 (2010).

[30] M. W. Johnson et al., Nature (London) 473, 194 (2011).

[31] M. Grajcar, A. Izmalkov, S. H. W. van der Ploeg, S. Linzen, E. Ilichev, T. Wagner, U. Hübner, H. G. Meyer, A. Maassen van den Brink, S. Uchaikin, and A. M. Zagoskin, Phys. Rev. B 72, 020503 (2005).

[32] M. Leib and M. J. Hartmann, New J. Phys. 12, 093031 (2010).

[33] M. I. Makin, J. H. Cole, C. D. Hill, A. D. Greentree, and L. C. L. Hollenberg, Phys. Rev. A 80, 043842 (2009).

[34] J. Koch and K. LeHur, Phys. Rev. A 80, 023811 (2009).

[35] S. Schmidt, D. Gerace, A. A. Houck, G. Blatter, and H. E. Türeci, Phys. Rev. B 82, 100507 (2010).

[36] S. Sachdev, Quantum Phase Transitions (Cambridge University Press, Cambridge, 2011).

[37] M. Scala, B. Militello, A. Messina, S. Maniscalco, J. Piilo, and K. Suominen, J. Phys. A: Math. Theor. 40, 14527 (2007).

[38] G. M. Reuther, D. Zueco, F. Deppe, E. Hoffmann, E. P. Menzel, T. Weissl, M. Mariantoni, S. Kohler, A. Marx, E. Solano et al., Phys. Rev. B 81, 144510 (2010). 